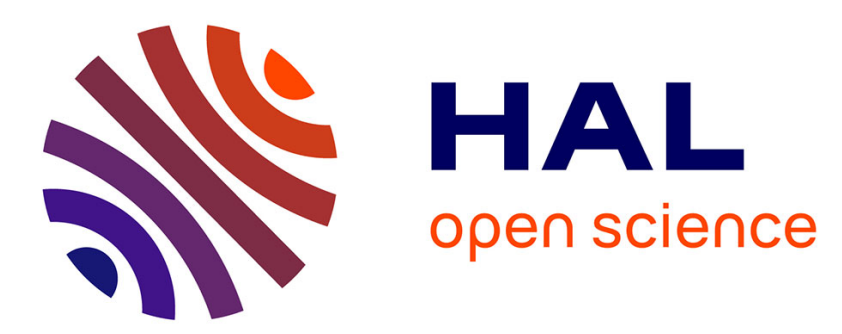

\title{
A cascaded loop structure in force and position to control a bilateral teleoperation robotic system
}

Pierre-Philippe Robet, Yannick Aoustin, Maxime Gautier, Sylvain Devie

\section{To cite this version:}

Pierre-Philippe Robet, Yannick Aoustin, Maxime Gautier, Sylvain Devie. A cascaded loop structure in force and position to control a bilateral teleoperation robotic system. 6th international conference on control, decision and information technologies, May 2019, Paris, France. hal-02362505

\section{HAL Id: hal-02362505 \\ https://hal.science/hal-02362505}

Submitted on 13 Nov 2019

HAL is a multi-disciplinary open access archive for the deposit and dissemination of scientific research documents, whether they are published or not. The documents may come from teaching and research institutions in France or abroad, or from public or private research centers.
L'archive ouverte pluridisciplinaire $\mathbf{H A L}$, est destinée au dépôt et à la diffusion de documents scientifiques de niveau recherche, publiés ou non, émanant des établissements d'enseignement et de recherche français ou étrangers, des laboratoires publics ou privés. 


\title{
A cascaded loop structure in force and position to control a bilateral teleoperation robotic system
}

\author{
Sylvain Devie, Pierre-Philippe Robet, Yannick Aoustin, and Maxime Gautier
}

\begin{abstract}
A cascaded force and position control is implemented on each of two robots of a bilateral teleoperation system. The aim is to propose a classical frequency approach in order to tune the closed-loop of each robot for different operating modes. Thus an efficient four channel bilateral teleoperation controller is proposed. It can be decomposed into four different two channel controller variations that can introduce inner virtual flexibility. This virtual flexibility is calculated for each case and can be tuned with the gains of the position control of the robots. An original way to calculate the apparent impedance is provided.
\end{abstract}

\section{INTRODUCTION}

Controlling the trajectory of a robot and its interaction forces with the environment is a challenging field in robotics. This mechatronic problem needs an accurate model of the full system: robot and controller ([1]). This paper focuses on the implementation of a bilateral teleoperation system, where an operator manipulates a master robot reproducing the behavior of a slave robot interacting with the environment. In this case the model of the system, which is composed of the master and slave robots, is particularly important to ensure the stability of the teleoperation task.

This problem is not a recent one. In [2] the author gives a good overview of the state of the art about the teleoperated systems during the nineties. Two main approaches can be cited. In [3], Lawrence defines a hybrid matrix that allows us to express the velocity of the master robot and the applied force by the operator on it according to the slave robot and its applied force to the environment. Then, this matrix is used to extract the transparency criterion and a stability condition thanks to the passivity theorem. Hannaford [4] defines another hybrid matrix allowing us to express the velocity of the end-effector of the first robot and the effort that a coupled second robot exchanges with its environment as a function of the velocity of the second robot and the effort exchanged by the first one with its environment. This model allows us to easily express the performance of the system. These two matrices can be easily derived from the calculations presented in this paper in order to extend our work with these stability and transparency criteria. The issue of transparency in time-delayed teleoperation is studied in [5]. A local force feedback is introduced for enhanced stability and performance.

Over the years, several specific devices have been designed for teleoperation systems. However, this paper focuses on the

\footnotetext{
The Authors are with the Université de Nantes, LS2N, CNRS UMR 6004, 44321 Nantes, France (Sylvain.Devie, Pierre-philippe.Robet, Yannick.Aoustin, Maxime.Gautier) @univ-nantes.fr
}

use of standard industrial robots with force feedback. This solution can be considered for the choice of the slave robot, as shown in [6] for example. Here the aim is to also use this solution for the master arm. In this configuration, the external forces are usually measured thanks to a force sensor. In [7], the authors discuss the best place to integrate the force sensor between the base of the robot and its end-effector. According to this study, a force sensor placed at the end-effector of the robot allows a better rejection of the inner frictions, while placing the sensor at the base allows us to measure an effort from the environment at any point of the robot. These classical industrial robots have important friction and damping coefficients. A classical approach to ensure their stability is based on the passivity analysis ([8]). The main advantage of this analysis is that it allows a decomposition of one two-robot system into two one-robot systems ([9]). However, the aim of this paper is to define a teleoperation system that allows us to tune the controller using frequency analysis and the desired performances of each robot.

We consider two one degree of freedom (dof) prismatic robots. These experimental devices are convenient mechatronic systems to design complex control in force and position as recently shown in [10]. Yashiro uses also two on- primastic-link robot in [11]. There, an acceleration-based impedance control law is used in a teleoperation scenario for fast environmental stiffness estimations with a time delay. In [12] a bilateral teleoperation control is improved by considering a non-linear model for the perturbation observer.

One of the main issues of a teleoperated system is the time delay for the information transmission between two robots. In [13] a specific design is developed to deal with this issue. In [14] the transmission delay is supposed to be unknown and non-constant. However, here the electronic connections, the proximity of the two robots and the use of a single piece of hardware for the control part allows us to neglect this delay.

We focus on the definition of one four channel bilateral teleoperation controller, which will be decomposed into four two channel bilateral teleoperation controllers. Each robot is controlled according to either the position of the other or the force that it exchanges with its environment. According to [15], a classical two channel configuration based on the position of the robot has good stability, but poor force reflection. This force reflection is improved in the cases mixing the force and the position references, but the four channel configuration is far better. We show that the poor force reflection between the two robots in the two channel configuration is due to an inner virtual spring-damping 
system between the robots created by the controller. Here, the stiffness of the virtual spring between the two robots is calculated as a function of the apparent stiffness of each robot, which is expressed as a function of their control law.

Here, each robot is controlled thanks to a cascaded loop controller, which is an improved version of the one used in our previous studies ([16], [10]). These studies showed an efficient control in force and velocity for single robot operations. Devie et al [16] showed how to tune the controller and focussed on the equivalence between this controller and an impedance controller [10].

This paper is outlined as follows: Section 2 describes the experimental setup and modeling. Section 3 presents our controllers and the associated calculations. Section 4 is devoted to simulation and experimental validation and section 5 offers our conclusion and perspectives.

\section{Modeling}

In the subsequent sections, the two similar robots are designated by subscript $i=1$ for the master robot and $i=2$ for the slave robot. Two EMPS (Electro-Mechanical Positioning System) robots are considered (Fig. 1). The main components of the EMPS robot are standard prismatic joint systems used in robot or machine tools. It is composed of a Maxon DC motor which drives a carriage, connected to a mass in translation by a high-precision low-friction ball screw. A flexible coupling can be used to connect the motor and the ball screw. The positions of the motor and the ball screw can be measured independently thanks to two incremental encoders equipping on the EMPS robots.

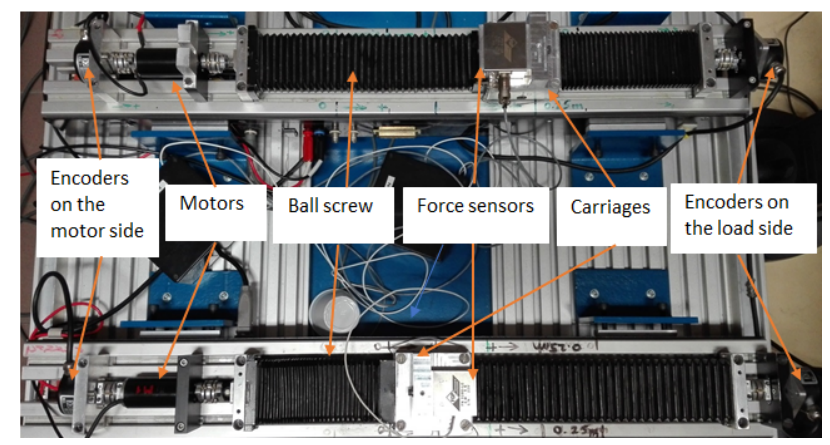

Fig. 1: EMPS robot main components

In the following, let us consider that the inner flexibility of the robot can be neglected when compared to that of the force sensor. Thus only the flexibility of the force sensor is considered. All variables are given in SI units on the load side.

The Maxon DC motor is driven thanks to an inner current loop, which can be considered as an equivalent gain $G_{I i}$ in the frequency range. Its torque constant is a known value $k_{t i}$ and it moves the load thanks to the ball screw, which has a reduction ration of $r_{i}$. We have the following linear relation between the reference voltage of the inner current loop $v_{I i}$ $(\mathrm{V})$ and the force from the motor on the load side $F_{m i}(\mathrm{~N})$ :

$$
F_{m i}=r_{i} \cdot k_{t i} \cdot G_{I i} \cdot v_{I i}=G_{\tau i} \cdot v_{I i}
$$

For the following study, the force sensor is fixed between the carriage and the tool interacting with the environment (Fig. 2). This sensor is a spring with a stiffness coefficient $K_{r i}$, which is given by the manufacturer and has been checked experimentally.

The considered mechanical system has two dof $\mathrm{s}$ : a rigid dof $q_{i}$ and a flexible dof $\delta_{i}$. The position $q_{i}$ of the end effector of the robot is measured and controlled by using an encoder sensor. The position $\delta_{i}$ is the relative deformation of the force sensor spring defined in order to have $F_{i}=0$ when $\delta_{i}=0, F_{i}$ is the force measured by the sensor. It defines the position $x_{i}$ of the end-effector such as:

$$
x_{i}=q_{i}+\delta_{i}
$$

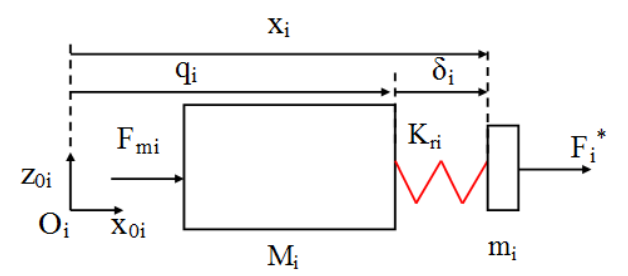

Fig. 2: Scheme of the moving part of the robot, the force sensor, and tool.

$M_{i}$ is the mass of carriage and the equivalent mass in translation of the motor and the screw inertia $(\mathrm{kg}), f_{v i}$ is the viscous damping coefficient $(N /(m / s))$ and $f_{c i}$ is the Coulomb friction $(N), m_{i}$ is the mass $(\mathrm{kg})$ of the force sensor and the tool. The friction forces on this body are neglected in front of the external forces.

With respect to the reference frame fixed to the robot, the dynamic model of the horizontal mechanical device is as follows:

$$
\begin{aligned}
& F_{m i}=M_{i} \ddot{q}_{i}+f_{v i} \dot{q}_{i}+f_{c i} \operatorname{sign}\left(\dot{q}_{i}\right)-K_{r i}\left(x_{i}-q_{i}\right) \\
& F_{i}^{*}=m_{i} \ddot{x}_{i}+K_{r i}\left(x_{i}-q_{i}\right)
\end{aligned}
$$

with $F_{m i}$ the actuation force on mass $M_{i}$ and $F_{i}^{*}$ the interaction force from the environment on mass $m_{i}$. In the following, let us differentiate the force $F_{i}^{*}$ applied on the system by the environment and the force $F_{i}=K_{r i}\left(x_{i}-q_{i}\right)$ measured by the force sensor.

The interaction force $F_{i}^{*}$ applied on the system and the velocity $\dot{x}_{i}$ are linked by the impedance of the environment: $Z_{e i}=F_{i}^{*} / \dot{x}_{i}(\mathrm{~s})$. This environment consists of the actions of the operator for the master robot and the reactions of the obstacle for the slave robot. In the latter case, the robot is controlled in order to apply a specific force on the environment, which has an impedance $Z_{e i}(\mathrm{~N} /(\mathrm{m} / \mathrm{s}))$. Three particular cases can be considered for the impedance. The softest case is the free case: $F_{i}^{*}=0$, so $Z_{e i}=0$, which means that there is no obstacle and the robot is free to move. The hardest case is the constraint case: $\dot{x}_{i}=0$, so $Z_{e i} \longrightarrow \infty$, which means that the environment is an infinitely rigid obstacle, the direct consequence is that $x_{i}$ is constant. In this case, the robot can be controlled by considering the flexibility of 
the force sensor, which gives the maximum stiffness of all components in series, force sensor and environment. The last case is an intermediate case, with an unknown environment, which is the case in most surgical applications.

\section{CONTROL DESIGN}

The control in force and position of one robot is firstly presented according to force and position references. These references are functions of the force and position measured on the other robot.

Then, the coupling between the robots will be presented and a mathematical study will define the expected behavior of the study.

\section{A. Focus on a single robot}

The considered controller is presented in Fig. 3 for one of the robots. In classical applications, we consider a light tool and the mass $m_{i}$ is neglected. The Coulomb friction $f_{c i}$ is assimilated to a constant perturbation, corrected by the integral action of the inner loop.

The considered controller uses an inner IP velocity loop allowing us to tune the dynamic behavior of the robot. The reference of this inner loop is the sum of the output of the feedback control in force and position, as shown in Fig. 3. The aim of these outer loops is to tune the apparent impedance of the robot around an equilibrium position.

In most applications, the outer force loop is far smaller than the force the motor has to produce to counter the dynamics of the system. The frequency range of this force is also ten times smaller than the bandwidth of the velocity loop. In these conditions, the influence of the external force may be neglected in the tuning of the velocity loop.

The open loop transfer function of the inner velocity loop is:

$$
\begin{aligned}
T_{b o \dot{q}_{r e f} \dot{q}_{i}}(\mathrm{~s}) & =\frac{\dot{q}_{i}(\mathrm{~s})}{\dot{q}_{i r e f}(\mathrm{~s})-\dot{q}_{i}(\mathrm{~s})} \\
& =\frac{k_{v i} G_{\tau i}}{t_{v i} \mathrm{~s}} \frac{1}{M_{i} \mathrm{~s}+f_{v i}+k_{v i} G_{\tau i}}
\end{aligned}
$$

Imposing $T_{b o \dot{q}_{r e f}} \dot{q}_{i}\left(\mathrm{j} \omega_{v}\right)=1 \mathrm{e}^{\mathrm{j}\left(-\pi+\phi_{v}\right)}$, leads us to tune $k_{v i}$ et $t_{v i}$. The closed loop transfer function of the inner velocity loop is:

$$
T_{b f \dot{q}_{i r e f} \dot{q}_{i}}(\mathrm{~s})=\frac{1}{1+\frac{2 z_{v i}}{\omega_{0 v i}} \mathrm{~s}+\frac{\mathrm{s}^{2}}{\omega_{0 v i}^{2}}}
$$

with $\omega_{0 v i}=\sqrt{K_{v i} / M_{i}}, z_{v i}=\left(t_{v i}+\frac{f_{v i}}{K_{v i}}\right) \frac{\omega_{0 v i}}{2}$ and $K_{v i}=\frac{k_{v i} G_{\tau}}{t_{v i}}$.

The stability and the performances of the outer loops can be calculated thanks to the open loop transfer functions in force and position, using the superposition principle. The open loop of the position controller can be easily calculated according to the open loop of the velocity controller. However, the external forces depend on the impedance of the environment. The open loop transfer function of the force controller is calculated in order to ensure a stable system in any case. In these conditions, around an equilibrium point (3) gives: $F_{i}^{*}=F_{i}=K_{r i} q_{i}$. The open outer loop transfer functions are:

$$
\begin{aligned}
& T_{b o q_{i r e f} q_{i}}(\mathrm{~s})=\frac{q_{i}(\mathrm{~s})}{q_{\text {iref }}(\mathrm{s})-q_{i}(\mathrm{~s})}=\frac{k_{p i} / \mathrm{s}}{1+\frac{2 z_{v i}}{\omega_{0 v i}} \mathrm{~s}+\frac{\mathrm{s}^{2}}{\omega_{0 v i}^{2}}} \\
& T_{b o F_{\text {iref }} F_{i}}(\mathrm{~s})=\frac{F_{i}(\mathrm{~s})}{F_{\text {iref }}(\mathrm{s})-F_{i}(\mathrm{~s})}=\frac{K_{r i} k_{e i} / \mathrm{s}}{1+\frac{2 z_{v i}}{\omega_{0 v i}} \mathrm{~s}+\frac{\mathrm{s}^{2}}{\omega_{0 v i}^{2}}}
\end{aligned}
$$

These two loops can be tuned in order to have the same bandwidth and tacking into account the worst case $\left(Z_{e i} \longrightarrow\right.$ $\infty)$.

Let us now calculate the apparent impedance of robot i alone. According to Fig. 3, we can write the following equation:

$$
\begin{aligned}
F_{m i}(\mathrm{~s}) & =G_{\tau i}\left(-k_{v i} \dot{q}_{i}(\mathrm{~s})-\frac{k_{v i}}{t_{v i} \mathrm{~s}} \dot{q}_{i}(\mathrm{~s})\right. \\
& +\frac{k_{e i} k_{v i}}{t_{v i} \mathrm{~s}}\left(-F_{i r e f}(\mathrm{~s})+F_{i}(\mathrm{~s})\right) \\
& \left.+\frac{k_{p i} k_{v i}}{t_{v i} \mathrm{~s}}\left(q_{i}(\mathrm{~s})-q_{r e f}(\mathrm{~s})\right)\right) \\
& =\left(M_{i} \mathrm{~s}+f_{v i}\right) \dot{q}_{i}(\mathrm{~s})-F_{i}(\mathrm{~s})
\end{aligned}
$$

The apparent impedance is calculated around an equilibrium position. This assumption leads us to choose:

- $F_{\text {iref }}=0$ and $q_{\text {iref }}=0$

The apparent impedance of the robot is:

$$
Z_{a i}=\frac{F_{i}(\mathrm{~s})}{\dot{q}_{i}(\mathrm{~s})}=\frac{M_{i} \mathrm{~s}+\left(f_{v i}+k_{v i} G_{\tau i}\right)+G_{\tau i} \frac{k_{v i}}{t_{v i} \mathrm{~s}}+G_{\tau i} \frac{k_{p i} k_{v i}}{t_{v i} \mathrm{~s}^{2}}}{1+G_{\tau i} \frac{k_{e i} k_{v i}}{t_{v i} \mathrm{~s}}}
$$

Assuming the bandwidth of the outer loop to be small compared to $G_{\tau i} \frac{k_{e i} k_{v i}}{t_{v i}}$ and neglecting the influence of the term $M_{i} s^{2}$ ([10]), we can write:

$$
Z_{a i}=\left(\frac{f_{v i}}{G_{\tau i}}+k_{v i}\right) \frac{t_{v i}}{k_{e i} k_{v i}} \mathrm{~s}+\frac{1}{k_{e i}}+\frac{k_{p i}}{k_{e i} \mathrm{~s}}=M_{a i} \mathrm{~s}+b_{a i}+\frac{K_{a i}}{\mathrm{~s}}
$$

In the following, $M_{a i}, b_{a i}$, and $K_{a i}$ will be used in the calculation of the coupled system. According to the definitions of these coefficients, the outer force loop can be used to tune the apparent mass and damping of the system. Then the outer position loop can be used to tune the apparent stiffness of the system.

\section{B. Four channel bilateral teleoperation}

Let us now consider the coupling of the two robots. Each robot is controlled thanks to the controller defined in Fig. 3. Between the two robots there is a four-channel bilateral coupling, see Fig. 4. Each bloc "robot" includes the controller and the mechanism as in Fig. 3. The teleoperated system is defined considering that the operator applies an effort $F_{1}$ to the system and follows the position $q_{1}$ of the master robot. The slave robot is in contact with the environment, which has an impedance $Z_{e}$. The interaction 


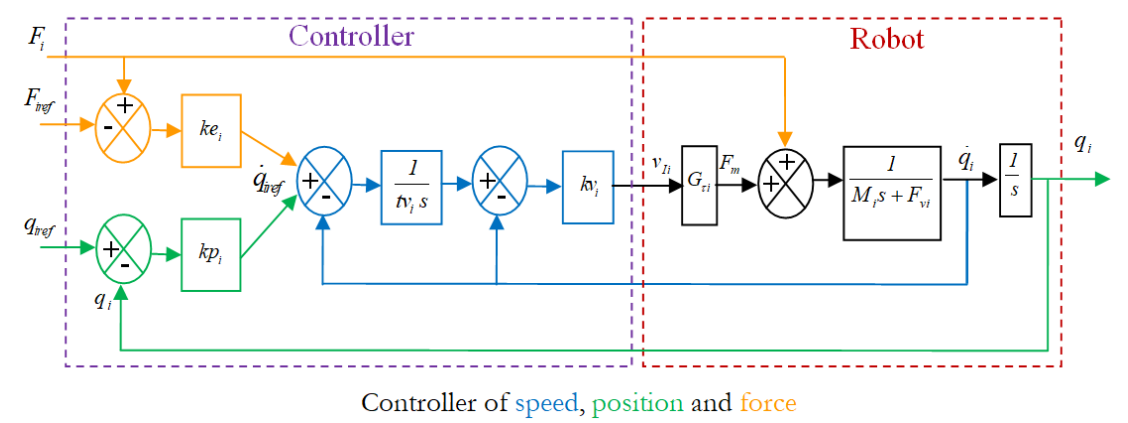

Fig. 3: Controller of speed (blue), position (green) and force (orange) for each robot

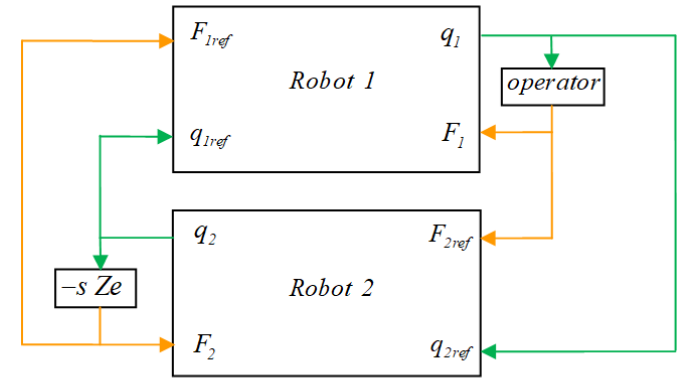

Four channel bilateral teleoperation

Fig. 4

between the robot and the environment is performed via a force sensor having a stiffness $K_{r 2}$. In this condition, the maximal stiffness of the group \{environment + sensor\} is $K_{r 2}$.

According to these hypotheses, $\frac{F_{2}(\mathrm{~s})}{\dot{q}_{2}(\mathrm{~s})}$ is the impedance of the environment. It should be the apparent impedance of robot 2 if its correction gains are well calculated. The reference of the controller of robot 1 depends on the measurements from the sensors of robot 2 . However, the reference of the controller of robot 2 depends on the measurements from the sensors of robot 1 . The consequence of this loop is that the impedance felt by the operator is no longer $Z_{a 1}$. The operator feels an impedance $Z_{a 1}^{c}$ for the coupled system, which depends on the apparent impedance of each robot and how the system is coupled. This impedance of the coupled system has to be calculated in order to tune the transparency of the system.

Based on Figs. 1 and 3 and similarly to [3] and [4], the linear control system of the two robots can be written under the following form:

$$
\begin{aligned}
& A_{1}(\mathrm{~s}) F_{1}(\mathrm{~s})-B_{1}(\mathrm{~s}) F_{r e f 1}(\mathrm{~s})=C_{1}(\mathrm{~s}) \dot{q}_{1}(\mathrm{~s})-D_{1}(\mathrm{~s}) \dot{q}_{\text {ref } 1}(\mathrm{~s}) \\
& A_{2}(\mathrm{~s}) F_{2}(\mathrm{~s})-B_{2}(\mathrm{~s}) F_{r e f 2}(\mathrm{~s})=C_{2}(\mathrm{~s}) \dot{q}_{2}(\mathrm{~s})-D_{2}(\mathrm{~s}) \dot{q}_{r e f 2}(\mathrm{~s})
\end{aligned}
$$

with

$$
\begin{aligned}
A_{i}(\mathrm{~s}) & =1+\frac{K_{v i} k_{e i}}{\mathrm{~s}}, B_{i}(\mathrm{~s})=\frac{K_{v i} k_{e i}}{\mathrm{~s}}, \\
C_{i}(\mathrm{~s}) & =M_{i} \mathrm{~s}+f_{v i}+M_{a i} k_{e i} K_{v i}+\frac{B_{a i} K_{v i} k_{e i}}{\mathrm{~s}}+\frac{K_{a i} K_{v i} k_{e i}}{\mathrm{~s}^{2}}, \\
& =M_{i} \mathrm{~s}+f_{v i}+Z_{a i}(\mathrm{~s}) \frac{k_{e i} K_{v i}}{\mathrm{~s}}
\end{aligned}
$$

According to these equations, it is possible to define a mathematical formulation of the apparent impedance of the coupled system with $F_{2}(\mathrm{~s})=-Z_{e} \dot{q}_{2}(\mathrm{~s})$. For the four bilateral teleoperation with $F_{r e f 1}(\mathrm{~s})=-F_{2}(\mathrm{~s})$ and $F_{r e f 2}(\mathrm{~s})=-F_{1}(\mathrm{~s})$, it comes:

$Z_{a 1}^{c}(\mathrm{~s})=\frac{C_{1}(\mathrm{~s})\left(A_{2}(\mathrm{~s}) Z_{e}(\mathrm{~s})+C_{2}(\mathrm{~s})\right)+D_{2}(\mathrm{~s})\left(Z_{e}(\mathrm{~s}) B_{1}(\mathrm{~s})-D_{1}(\mathrm{~s})\right)}{A_{1}(\mathrm{~s})\left(A_{2}(\mathrm{~s}) Z_{e}(\mathrm{~s})+C_{2}(\mathrm{~s})\right)-B_{2}(\mathrm{~s})\left(Z_{e}(\mathrm{~s}) B_{1}(\mathrm{~s})-D_{1}(\mathrm{~s})\right)}$

This equation is quite complex and does not allow us to calculate a clear equation of all the mechanical parameters of the apparent impedance of the coupled system. However, considering the steady state, it is possible to calculate the apparent stiffness felt by the operator when $Z_{e}(\mathrm{~s})=\frac{K_{e}}{\mathrm{~s}}$ with $K_{e}$ the stiffness or the sensor+environment:

$$
K_{a 1}^{c}=\frac{K_{a 1} K_{a 2}+K_{a 1} K_{e}+K_{a 2} K_{e}-K_{a 1} K_{a 2}}{K_{a 1}+K_{a 2}}=K_{e}
$$

According to this result, the four channel bilateral teleoperation system is transparent in steady state: the operator feels the stiffness of the environment and not that of the robot. However, the four channel configuration can be power consuming, especially for multibody systems. In these conditions, it might be better to use a two channel bilateral teleoperation system.

\section{Two channel bilateral teleoperation}

Based on the previous study, a two channel bilateral teleoperation system can be defined by limiting the interaction between the two robots. In the preceding section, each robot is controlled according to the position of the other robot as well as the force it exchanges with its environment. However, here each robot will be controlled with only one of these channels of information.

If the robot $i$ is controlled thanks to the effort of the other robot, the force reference $F_{\text {iref }}$ will be defined, but not the position reference $q_{\text {iref }}$. Then, the outer position loop will create a virtual stiffness between the robot and its reference position $q_{i r e f}$. In this case, it is possible to define this reference position as a function of the effort or to suppress it. In the following, this last solution will be used. It implies that $k_{p i}=0$ and the direct consequence is $D_{i}(\mathrm{~s})=0$ into (10) and $K_{a i}=0$.

If the robot $i$ is controlled thanks to the position of the other robot, the position reference $q_{\text {iref }}$ will be defined, but 
not the force reference $F_{\text {iref }}$. However, if the master arm is controlled without the force loop, the operator will have to compensate for the dynamics of the system. This operation is possible for robots with specific designs, but most systems have a large gear ratio, which can limit its action. This problem is also valid in the case of a slave robot. In the following, it is solved by keeping an open force loop with $F_{\text {iref }}=0$.

Four cases are considered and named based on which information channel is used for the control of each robot: position $(\mathrm{P})$ or effort $(\mathrm{F})$, starting from robot 1. For example, in the case $\mathrm{PF}$, robot 1 is controlled thanks to the position of robot 2, which is controlled thanks to the effort that the operator applies on robot 1 .

Similar to the previous section, the apparent stiffness of robot 1 can be calculated for the teleoperation robotic system. The results of this calculation are shown in table I for the four considered cases.

TABLE I: Calculation of the apparent stiffness $K_{a 1}^{c}$ in four cases of the two channel bilateral teleoperation system

\begin{tabular}{|c|c|c|}
\hline Robot 2 & $\mathrm{~F}$ & $P$ \\
\hline $\mathrm{F}$ & $K_{e}$ & $\frac{K_{a 1} K_{e}}{K_{a 1}+K_{e}}$ \\
\hline$P$ & $\frac{K_{a 2} K_{e}}{K_{a 2}+K_{e}}$ & $\frac{K_{a 1} K_{e}}{K_{a 2}+K_{e}}$ \\
\hline
\end{tabular}

According to the results presented in Table I, the apparent stiffness in the case of the FF controller is exactly that of the environment. This result was expected because, without the position loop, none of the robots have an apparent stiffness when it is considered independent of the other one. In the case of a PP controller, the apparent stiffness depends on the apparent stiffness of the two robots. Thus, the transparency of the system can be increased by increasing the two apparent stiffnesses $K_{a 1}$ and $K_{a 2}$, with $K_{a 1}=K_{a 2}$. In the hybrid cases $\mathrm{PF}$ and FP, the apparent stiffness of the coupled system is equivalent to the stiffness of the environment in series with the stiffness of the robot controlled according to the position of the other one. Thus, the transparency of the system can be increased by increasing the apparent stiffness of the robot. It is important to notice that the stiffness of the environment is limited by one of the force sensors, so the apparent stiffness of the robot can be higher than the environmental stiffness even when the robot is in contact with a rigid obstacle. However, increasing the apparent stiffness $K_{a i}$ can be done by increasing the gain $k_{e i}$ of the position loop, which can lead to instability, or by decreasing the gain $k_{p i}$, which increases the apparent mass and the apparent damping of the system.

\section{EXPERIMENTAL RESULT}

Experiments have proven the efficiency of the presented controllers. However, in order to compare it, the operator should apply exactly the same effort on the robot for each controller, with the exact same environment. In this section, a simulation will firstly be performed in order to compare different controllers and the most efficient one will be chosen for experimental validation.

\section{A. Simulation result}

Some simulations are performed with the two EMPS robots considering the real values of the arms. These robots were identified for this study [17]. The parameters used in the previous section are: $M_{1}=105 \mathrm{~kg}, f_{v 1}=313 \mathrm{~N} /(\mathrm{m} / \mathrm{s})$, $f_{c 1}=13 \mathrm{~N}, G_{\tau 1}=35 \mathrm{~N} / \mathrm{V}$ and $K_{r 1}=210^{4} \mathrm{~N} / \mathrm{m}$ for the master robot and $M_{2}=102 \mathrm{~kg}, f_{v 2}=340 \mathrm{~N} /(\mathrm{m} / \mathrm{s}), f_{c 2}=$ $18 \mathrm{~N}, G_{\tau 2}=35 \mathrm{~N} / \mathrm{V}$ and $K_{r 2}=410^{4} \mathrm{~N} / \mathrm{m}$ for the slave robot.

The five considered control laws are tested for a trapezoidal force trajectory from the operator's side. It is assumed that the environmental impedance is just a stiffness $K_{e}=10^{4} \mathrm{~N} / \mathrm{m}$. For each experiment, we consider the trajectory of the forces exchanged between the two robots and their environment, and the position trajectories of both robots. The root mean square errors between these trajectories are calculated and presented in table II.

TABLE II: RMS error (\%) in position, effort and velocity for a trapezoid trajectory of the operator effort, for all the considered controllers

\begin{tabular}{|c||l|l|}
\hline Controller & $\begin{array}{l}\text { Position } \\
(\%)\end{array}$ & $\begin{array}{l}\text { Effort } \\
(\%)\end{array}$ \\
\hline four channel & $1.510^{-4}$ & 3 \\
two channel FF & $7.110^{-1}$ & 3.5 \\
two channel FP & $7.310^{1}$ & 7 \\
two channel PF & $7.310^{1}$ & 3.5 \\
two channel PP & $7.310^{1}$ & 10 \\
\hline
\end{tabular}

According to these results, the most efficient controller is the one using four channels and it ensures efficient following of the trajectories of force and position. For the two channel configurations, the most efficient controller is the FF controller, which allows good force control thanks to its structure, and a good transparency because it allows the operator to feel the stiffness of the environment. In the other two channel configurations, the apparent stiffness strongly depends on the position correction. This dependency is equivalent to a virtual spring between the two robots, which influences the position trajectory leading to large tracking errors. But this effect of a virtual spring is researched for some specific applications.

The stiffness of this virtual spring is calculated for the four cases in table I. It can depend on the apparent stiffness of the master robot, the slave robot or both of them. This calculation allows the operator to choose the configuration according to the robot to be tuned.

\section{B. Experimental result}

The most efficient controller is tested in the experimental test-beds. This experimentation is done with the four channel bilateral teleoperation control law. Both robots are controlled thanks to an input/output DSpace card. A single system is controlling both robots, which are located in the same small area, minimizing the time delay. This minimization 
is good enough to consider no time delay issue during the experiments.

The experiments are performed with the following protocol: the operator applies a specific effort on robot 1 while maintaining contact. The robot 2 is in contact with a stiff environment. This passive environment can push the robot when it is in contact, but not pull it. Two external force sensors are placed on the end-effector of the robot, measuring the efforts of the operator and the stiff environment. And there is no problem of discretization because the sampling rate of the system is 100 times higher than the working frequency.

The result of this experiment is presented in Fig. 5.
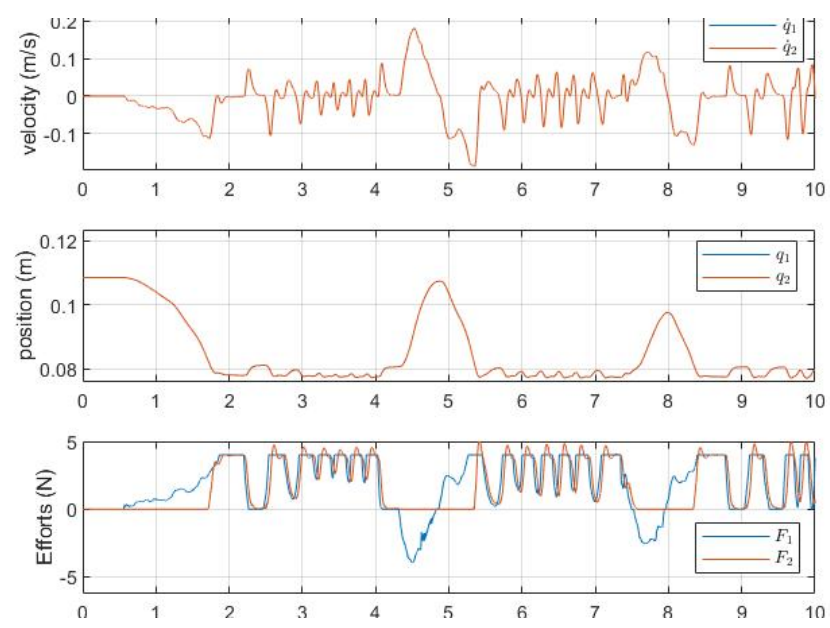

Fig. 5: Result of the experiments for four channel bilateral teleoperation controller

This figure shows a good tracking of the position and velocity between the two robots with no visible error. The RMS error of the position tracking is equal to $10^{-2} \%$ of the maximal displacement. However, the tracking RMS error of force is equal to $38 \%$ of the maximal force applied to the system. This error is mostly due to the fact that the environment can only push the robot 2 . Actually, because of the good position tracking, the robot can move with no contact with the environment. In this case, it does not exchange any force and the error tracking is not relevant. These configurations can easily be identified in Fig. 5 because the force $F_{2}$ is equal to $0 \mathrm{~N}$, for example at the beginning of the experiment, before the robot 2 is in contact with the environment before $1.8 \mathrm{~s}$, or at around $4.3 \mathrm{~s}$, when the operator pulls the robot 1 and removes the contact between the two robots.

If only the contact configuration is considered, the RMS error of the force tracking decreases to $17 \%$ of the maximal value of the exchanged force. Other experiments are performed with a two channel bilateral teleoperation controller and allowed the operator to tune the apparent stiffness of the coupled system and feel it when the master robot is manipulated.

\section{CONCLUSION}

A simple and efficient controller is proposed for the four channel bilateral teleoperation. This controller presents an inner velocity loop which can be tuned in order to have similar dynamics for the two robots. Then two outer force and position loops can be tuned in order to adapt to the apparent impedance of the system.

Different two channel bilateral teleoperation controllers are defined from this controller and are allowed to tune the impedance felt by the operator when the master arm is manipulated. This tuning allows the correction of the transparency of the system. The main contribution of this paper is mainly the statement of the apparent impedance given by (11).

\section{REFERENCES}

[1] K. Ohnishi, M. Shibata, and T. Murakami, "Motion control for advanced mechatronics," IEEE/ASME Trans. on Mechatronics, vol. 1, no. 1, pp. 56-67, 1996.

[2] T. B. Sheridan, "Teleoperation, telerobotics and telepresence: A progress report," Control Engineering Practice, vol. 3, no. 2, pp. 205214, 1995.

[3] D. A. Lawrence, "Stability and Transparency in Bilateral Teleoperation," IEEE Trans. on Robotics and Automation, vol. 9, no. 5, pp. 624637, 1993.

[4] B. Hannaford, "A Design Framework for Teleoperators with Kinesthetic Feedback," IEEE Trans. on Robotics and Automation, vol. 5, no. 4, pp. 426-434, 1989.

[5] K. Hashtrudi-Zaad and S. E. Salcudean, "Transparency in TimeDelayed Systems and the Effect of Local Force Feedback for Transparent Teleoperation," IEEE Trans. on Robotics and Automation, vol. 18, no. 1, pp. 108-114, 2002.

[6] G. Piolain, F. Geffard, A. Coudray, P. Garrec, J. F. Thro, and Y. Perrot, "Dedicated and standard industrial robots used as force-feedback telemaintenance remote devices at the AREVA recycling plant," Int. Conf. on Applied Robotics for the Power Industry, CARPI 2010, pp. 16, 2010.

[7] F. Geffard, C. Andriot, A. Micaelli, and G. Morel, "On the use of a base force/torque sensor in teleoperation," IEEE Int. Conf. on Robotics and Automation., vol. 3, no. April, pp. 2677-2683, 2000.

[8] B. E. Miller, J. E. Colgate, and R. A. Freeman, "On the Role of Dissipation in Haptic Systems," IEEE Trans. on Robotics, vol. 20, no. 4, pp. 768-771, 2004.

[9] D. Lee and P. Y. Li, "Passive bilateral control and tool dynamics rendering for nonlinear mechanical teleoperators," IEEE Trans. on Robotics, vol. 21, no. 5, pp. 936-951, 2005.

[10] S. Devie, P.-p. Robet, Y. Aoustin, and M. Gautier, "Impedance control using a cascaded loop force control .," IEEE Robotics \& Automation letter, pp. 1-7, 2018.

[11] D. Yashiro, "Fast Stiffness Estimation using Acceleration-based Impedance Control and its Application to Bilateral Control," in IFAC World Congress, pp. 12565-12570, 2017.

[12] K. Seki, S. Fujihara, and M. Iwasaki, "Improvement of Force Transmission Performance Considering Nonlinear," in IFAC World Congress, pp. 12577-12582, 2017.

[13] J. Guo, C. Liu, and P. Poignet, "Stable and enhanced position-force tracking for bilateral teleoperation with time delay," European Control Conference,, pp. 1980-1985, 2015.

[14] R. J. Anderson and M. W. Spong, "Bilateral Control of Teleoperators with Time Delay," IEEE Trans. on Automatic Control, vol. 34, no. 5, pp. 494-501, 1989.

[15] I. Aliaga, A. Rubio, and E. Sanchez, "Experimental Quantitative Comparison of Different Control Architectures for Master-Slave Teleoperation," IEEE Trans. on Control Systems Technology, vol. 12, no. 1, pp. 2-11, 2004.

[16] S. Devie, P. P. Robet, Y. Aoustin, M. Gautier, and A. Jubien, "Accurate force control and co-manipulation control using hybrid external command," IFAC World Congress, pp. 2271-2276, 2017.

[17] M. Gautier, A. Jubien, A. Janot, and P. P. Robet, "Dynamic Identification of flexible joint manipulators with an efficient closed loop output error method based on motor torque output data," in IEEE Int. Conf. on Robotics and Automation, pp. 2949-2955, 2013. 